\title{
ON THE GENERALIZED BENJAMIN-ONO EQUATION
}

\author{
CARLOS E. KENIG, GUSTAVO PONCE, AND LUIS VEGA
}

\begin{abstract}
We study well-posedness of the initial value problem for the generalized Benjamin-Ono equation $\partial_{t} u+u^{k} \partial_{x} u-\partial_{x} D_{x} u=0, k \in \mathbb{Z}^{+}$, in Sobolev spaces $H^{s}(\mathbb{R})$. For small data and higher nonlinearities $(k \geq 2)$ new local and global (including scattering) results are established. Our method of proof is quite general. It combines several estimates concerning the associated linear problem with the contraction principle. Hence it applies to other dispersive models. In particular, it allows us to extend the results for the generalized Benjamin-Ono to nonlinear Schrödinger equations (or systems) of the form $\partial_{t} u-i \partial_{x}^{2} u+P\left(u, \partial_{x} u, \bar{u}, \partial_{x} \bar{u}\right)=0$.
\end{abstract}

\section{INTRODUCTION}

The purpose of this paper is to study the initial value problem (IVP) for the generalized Benjamin-Ono (B-O) equation

$$
\begin{gathered}
\partial_{t} u+u^{k} \partial_{x} u-\partial_{x} D_{x} u=0, \quad t, x \in \mathbb{R}, k \in \mathbb{Z}^{+}, \\
u(x, 0)=u_{0}(x),
\end{gathered}
$$

where $D_{x}=\left(-\partial_{x}^{2}\right)^{1 / 2}$.

In the case $k=1$ the equation in (1.1) was deduced by Benjamin [3] and Ono [28] as a model in internal-wave theory. Higher order nonlinearities (specially the case $k=2$ ) also appear in applications [6]. The generalized B-O equation presents the interesting fact that the dispersive effect is described by a nonlocal operator and is weaker than that exhibited by the generalized Korteweg-de Vries equation

$$
\partial_{t} u+u^{k} \partial_{x} u+\partial_{x}^{3} u=0
$$

Several works have been devoted to the existence problem for solutions of (1.1) with data $u_{0} \in H^{s}(\mathbb{R})=\left(1-\partial_{x}^{2}\right)^{-s / 2} L^{2}(\mathbb{R})$. In this direction the strongest results can be gathered in the following theorem.

Theorem 1.1. (i) Let $u_{0} \in H^{s}(\mathbb{R})$ with $s=0$ or $s=\frac{1}{2}$. Then for $k=1$ or $k=2$ there exists a (weak) solution $u$ of (1.1) such that

$$
u \in L^{\infty}\left(\mathbb{R}: H^{s}\right) \cap C_{w}\left(\mathbb{R}: H^{s}\right) \cap L_{\mathrm{loc}}^{2}\left(\mathbb{R}: H_{\mathrm{loc}}^{s+1 / 2}\right) .
$$

Received by the editors July 17, 1991.

1991 Mathematics Subject Classification. Primary 35Q30; Secondary 35G25, 35D99.

Key words and phrases. Generalized Benjamin-Ono equation, initial value problem, well-posedness. 
(ii) Let $u_{0} \in H^{1}(\mathbb{R})$. Then for $k=1$ there exists a (weak) solution $u$ of (1.1) such that

$$
u \in C_{b}\left(\mathbb{R}: L^{2}\right) \cap L^{\infty}\left(\mathbb{R}: H^{1}\right) \cap L_{\mathrm{loc}}^{2}\left(\mathbb{R}: H_{\mathrm{loc}}^{3 / 2}\right) .
$$

(iii) Let $u_{0} \in H^{3 / 2}(\mathbb{R})$. Then for $k=1$ the IVP (1.1) has a unique (strong) solution $u$ satisfying

$$
u \in C_{b}\left(\mathbb{R}: H^{3 / 2}\right) \cap L_{\text {loc }}^{2}\left(\mathbb{R}: H_{\text {loc }}^{2}\right) \cap L_{\text {loc }}^{4}\left(\mathbb{R}: L_{1}^{\infty}\right) \equiv X .
$$

Moreover the map $u_{0} \rightarrow u(t)$ from $H^{3 / 2}(\mathbb{R})$ to $X$ is continuous.

(iv) Let $u_{0} \in H^{s}(\mathbb{R})$ with $s>\frac{3}{2}$. Then for any $k \in \mathbb{Z}^{+}$there exist $T=$ $T\left(\left\|u_{0}\right\|_{s, 2} ; k\right)>0$ and a unique (strong) solution $u$ of (1.1) satisfying

$$
u \in C\left([-T, T]: H^{s}\right) \cap L_{\mathrm{loc}}^{2}\left([-T, T]: H_{\mathrm{loc}}^{s+1 / 2}\right) \equiv Y_{T} .
$$

Moreover given $T^{\prime} \in(0, T)$ there exists a neighborhood $U_{u_{0}}$ of $u_{0}$ in $H^{s}$ such that the map $\tilde{\bar{u}}_{0} \rightarrow \widetilde{\bar{u}}(t)$ from $U_{u_{0}}$ to $Y_{T^{\prime}}$ is continuous. When $k=1$ this result extends to any time interval.

Above we have used the notation:

$$
\begin{aligned}
C_{w}(\mathbb{R}: B)= & \text { the space of all weakly continuous function } \\
& \text { on } \mathbb{R} \text { to } B \text { (Banach space), }
\end{aligned}
$$

and

$$
C_{b}(\mathbb{R}: B) \equiv C(\mathbb{R}: B) \cap L^{\infty}(\mathbb{R}: B) .
$$

Part (i) of Theorem 1.1 is due to Ginibre and Velo [16]. Part (ii) was proven by Ginibre and Velo [16] and Tom [40]. Result (iii) was established by Ponce [30]. Finally (iv) follows by combining the works of Iorio [18], Abdelouhab, et al. [1], and Ponce [29] (for related results see [32, 21]).

From Theorem 1.1 we see that for $k>2$ and $u_{0} \in H^{s}(\mathbb{R})$ with $s \leq \frac{3}{2}$ the existence problem for (1.1) is open. On the other hand the proof of the local well-posedness result for (1.1) in $H^{s}$ with $s>\frac{3}{2}$ (Theorem 1.1(iv)) does not use the dispersive structure of the equation.

Also Theorem 1.1 tells us that the smoothing effect of Kato type [20] established in solutions of (1.1) is weaker (even locally in time) than that deduced by Kenig, Ponce, and Vega [23] in solutions of the associated linear problem. More precisely, if $\{V(t)\}_{-\infty}^{\infty}$ denotes the unitary group associated to the linear problem

$$
\begin{gathered}
\partial_{t} u-\partial_{x} D_{x} u=0, \quad x, t \in \mathbb{R}, \\
u(x, 0)=u_{0}(x),
\end{gathered}
$$

i.e., $u(x, t)=V(t) u_{0}(x)$, then it was shown in [20, Theorem 4.1] that there exists $c>0$ such that for each $x \in \mathbb{R}$

$$
\left(\int_{-\infty}^{\infty}\left|D_{x}^{1 / 2} V(t) u_{0}(x)\right|^{2} d t\right)^{1 / 2}=c\left\|u_{0}\right\|_{2} .
$$

Observe that although the gain of derivatives in (1.4) (as in Theorem 1.1) is also equal to $\frac{1}{2},(1.4)$ describes a $L_{x}^{\infty} L_{t}^{2}$ estimate instead of the $L_{\text {loc }}^{2} L_{\text {loc }}^{2}$ one sees in Theorem 1.1 .

Our main results in this paper show that for $k \geq 2$ and small data the results in Theorem 1.1 can be significantly improved. 
Theorem 1.2. Let $k \geq 2$ and $s$ be such that

$$
\begin{array}{ll}
s>1 & \text { if } k=2, \\
s>\frac{5}{6} & \text { if } k=3, \\
s \geq \frac{3}{4} & \text { if } k \geq 4 .
\end{array}
$$

There exists $\delta=\delta(k)>0$ such that for any $u_{0} \in H^{s}(\mathbb{R})$ with $\left\|u_{0}\right\|_{s, 2} \leq \delta$ there exist $T=T\left(\left\|u_{0}\right\|_{s, 2} ; k\right)>0$ (with $T(\rho, k) \rightarrow \infty$ as $\rho \rightarrow 0$ ) and a unique (strong) solution $u(\cdot)$ of the IVP (1.1) satisfying $u \in C\left([-T, T]: H^{s}(\mathbb{R})\right)$ and

$$
\sup _{0 \leq \theta \leq 1}\left\|D_{x}^{\theta(s+1 / 2)} u\right\|_{L_{x}^{j /(1-\theta)} L_{T}^{2 / \theta}}<\infty
$$

where

$$
\|\omega\|_{L_{x}^{p} L_{T}^{q}}=\left(\int_{-\infty}^{\infty}\left(\int_{-T}^{T}|\omega(x, t)|^{q} d t\right)^{p / q} d x\right)^{1 / p}
$$

and $j=\min \{k ; 4\}$.

If $u_{0} \in H^{s^{\prime}}(\mathbb{R})$ with $s^{\prime}>s$ then the results above hold with $s^{\prime}$ instead of $s$ in the same time interval $[-T, T]$.

For $T^{\prime} \in(0, T)$ there exists a neighborhood $U_{u_{0}}$ of $u_{0}$ in $H^{s}(\mathbb{R})$ such that the map $\tilde{\bar{u}}_{0} \rightarrow \tilde{\bar{u}}(t)$ from $U_{u_{0}}$ to $X_{T^{\prime}, j}^{s}$ is Lipschitz where

$$
\begin{array}{r}
X_{T, j}^{s}=\left\{\omega: \mathbb{R} \times[-T, T] \rightarrow \mid \sup _{[-T, T]}\|\omega(t)\|_{s, 2}<\infty\right. \\
\text { and } \left.\sup _{0 \leq \theta \leq 1}\left\|D_{x}^{\theta(s+1 / 2)} \omega\right\|_{L_{x}^{j /(1-\theta)} L_{T}^{2 / \theta}}<\infty\right\} .
\end{array}
$$

Theorem 1.3. For $k \geq 4$ and $s \geq 1$ the results of Theorem 1.2 extend to the time interval $(-\infty, \infty)$. In addition, the solution $u$ satisfies

$$
u \in C_{b}\left(\mathbb{R}: H^{s}(\mathbb{R})\right),
$$

and

$$
\begin{gathered}
\left(\int_{-\infty}^{\infty}\|u(\cdot, t)\|_{\infty}^{4} d t\right)^{1 / 4}<\infty \\
\sup _{0 \leq \theta \leq 1}\left\|D_{x}^{\theta(s+1 / 2)} \omega\right\|_{L_{x}^{4 /(1-\theta)} L_{t}^{2 / \theta}}<\infty .
\end{gathered}
$$

(See notations at the end of this section).

As a consequence of Theorem 1.3 we obtain the following scattering type result (see [36]).

Corollary 1.4. Let $k \geq 4$ and $u_{0} \in H^{1}(\mathbb{R})$ satisfying the smallness assumption of Theorem 1.2. Then for the corresponding solution $u(t)$ of the IVP (1.1) there exist unique $\omega_{0^{ \pm}} \in H^{1}(\mathbb{R})$ such that

$$
\lim _{t \rightarrow \pm \infty}\left\|u(t)-V(t) \omega_{0 \pm}\right\|_{1,2}=0 .
$$

Remarks. (a) Since Theorem 1.2 deals with fractional derivatives in $L^{\infty}$ the use of the one parameter family of norms introduced in (1.6) is necessary, since $D^{i \gamma}$ is not bounded in $L^{\infty}$ and hence complex interpolation is not possible. 
Notice that for $\theta=1$ (1.6) provides the sharp version $\left(L_{x}^{\infty} L_{T}^{2}\right)$ of the Kato smoothing effect described in (1.3).

(b) Combining the result (iii) in Theorem 1.1 with those in Theorem 1.3 one sees that for $u_{0} \in H^{3 / 2}(\mathbb{R})$ with $\delta_{0}=\left\|u_{0}\right\|_{3 / 2,2} \ll 1$ the IVP (1.1) has a global solution for power $k=1,4,5, \ldots, j\left(\delta_{0}\right)$. To explain this gap we observe that in the case $k=1$ the global result is due to the conservation laws (see $[5,9])$, and holds for arbitrary data. On the other hand, the result for $k \geq 4$ is a consequence of the $L_{t}^{4} L_{x}^{\infty}$-estimate of Strichartz type [37, 15] satisfied by solutions of the associated linear problem. In particular $(1.7 \mathrm{a}-\mathrm{c})-(1.8)$ tell us that for small data in $H^{1}(\mathbb{R})$ the asymptotic behavior of the solution is controlled by the dispersive part, and hence scattering occurs.

Also worth noting are the works of Bona, Souganidis, and Strauss [7] and Weinstein [43] where the critical power for stability and instability of the solitary waves solutions of (1.1) was shown to be $k=2$. In the case of the generalized Korteweg-de Vries both critical values (i.e., that for small data scattering [26] and the one for the stability of the solitary waves [7, 43]) agree and are equal to 4 .

(c) The results in Theorem 1.2 could be extended to the limiting cases in (1.4) (i.e., $s=1$ if $k=2$ and $s=\frac{5}{6}$ if $k=3$ ) by proving the following estimate:

$$
\left(\int_{-\infty[-1,1]}^{\infty} \sup _{\mid}\left|V(t) u_{0}\right|^{2} d x\right)^{1 / 2} \leq c\left\|u_{0}\right\|_{1 / 2,2} .
$$

However we do not know whether or not inequality (1.9) is true.

(d) Our method of proof combines several estimates concerning the associated linear problem (1.2) with the contraction principle and the integral equation form of $(1.1)$

$$
u(t)=V(t) u_{0}-\int_{0}^{t} V(t-\tau)\left(u^{k} \partial_{x} u\right)(\tau) d \tau .
$$

The main new tool here is the inhomogeneous version of the smoothing effect of Kato type (1.3). We shall prove (see Theorem 2.1, estimate (2.5) below) that

$$
\left\|D_{x} \int_{0}^{t} V(t-\tau) F(\cdot, \tau) d \tau\right\|_{L_{x}^{\infty} L_{t}^{2}} \leq C\|F\|_{L_{x}^{1} L_{t}^{2}}
$$

(see notations at the end of this introduction).

Thus (1.11) affirms that the gain of derivatives in the inhomogeneous case is twice that described in (1.3) for the homogeneous problem. For further comments and references related to the estimate (1.11) and its extension to higher dimensions in the case of the free Schrödinger group see [25].

The same approach presented here (linear estimates and contraction principle) applies to other dispersive models. For example, combining the results below with some obtained in [26], the IVP for the generalized KdV equation (1.2) is locally well-posed in all $H^{s}(\mathbb{R})$ with

$$
\begin{aligned}
s>\frac{3}{4} & \text { if } k=1, \\
s \geq \frac{1}{4} & \text { if } k=2, \\
s \geq \frac{1}{12} & \text { if } k=3, \\
s \geq(k-4) / 2 k & \text { if } k \geq 4 .
\end{aligned}
$$


It easily follows that the IVP

$$
\begin{gathered}
\partial_{t} u+u^{k} \partial_{x} u-\partial_{x} D_{x}^{\mu} u=0, \quad t, x \in \mathbb{R}, \mu \in(1,2), \\
u(x, 0)=u_{0}(x),
\end{gathered}
$$

is locally well-posed in all $H^{s}(\mathbb{R})$ with $s \geq(9-3 \mu) / 4$ for $k=1$ (already proved in [24, Theorem 1.3]), $s \geq(7-3 \mu) / 4$ for $k=2, s \geq(19-9 \mu) / 12$ for $k=3$, and so on. In the case studied here $\mu=1$ the restriction on the size of the data appears since the gain of derivatives in (1.11) is equal to 1 (the amount of derivative in the nonlinear term). Thus to overcome the loss of derivatives in the integral equation (1.10) one needs to use the estimate (1.11) complemented with one for the maximal function (i.e., $\left.\sup _{-T \leq t \leq T}\left|V(t) u_{0}\right|\right)$. However, this quantity cannot be made arbitrarily small by taking $T$ small. When $\mu>1$ in (1.12) (higher dispersivity) one just needs to estimate $\left\|V(t) u_{0}\right\|_{L^{p}([-T, T])}$ with $p<\infty$ for which the necessary smallness hypothesis holds.

(e) It is easy to see that the group $\left\{e^{i t t_{x}^{2}}\right\}_{-\infty}^{\infty}$ satisfies similar estimates to those discussed in $\S 2$ for the group $\{V(t)\}_{-\infty}^{\infty}$ (see [25]). Also our method of proof works equally well for real or complex valued functions. Therefore the results in Theorems $1.2-1.3$ and Corollary 1.4 extend without major modifications to the IVP

$$
\begin{gathered}
\partial_{t} u=i \partial_{x}^{2} u+P_{1}(u, \bar{u}) \partial_{x} u+P_{2}(u, \bar{u}) \partial_{x} \bar{u}, \\
u(x, 0)=u_{0}(x),
\end{gathered}
$$

where $P_{j}: \mathbb{C}^{2} \rightarrow \mathbb{C}$ for $j=1,2$ are polynomials such that

$$
P_{j}\left(z_{1}, z_{2}\right)=\sum_{\alpha+\beta \geq 2} \alpha_{j \alpha \beta} z_{1}^{\alpha} z_{2}^{\beta} .
$$

In particular, when $P_{1}\left(z_{1}, z_{2}\right)=2 z_{1}, z_{2}$ and $P_{2}\left(z_{1}, z_{2}\right)=z_{1}^{2}$ we obtain an improvement in the case of small data of the results of Tsutsumi and Fukuda [41, Theorem 1].

It is interesting to remark that when the nonlinearity in (1.13) is replaced by a polynomial $P\left(u, \bar{u}, \partial_{x} u, \partial_{x} \bar{u}\right)$ with

$$
P\left(z_{1}, z_{2}, z_{3}, z_{4}\right)=\sum_{|\alpha| \geq 4} a_{\alpha} z^{\alpha}
$$

the results of Theorem 1.3 and Corollary 1.4 still hold in $H^{3}(\mathbb{R})$ and extend to systems. For this general nonlinearity (1.14) local well-posedness for the IVP (1.3) with small data was already established in [25] (in any dimension). Thus one has the following unusual situation: for small data global existence of classical solutions can be established, however, for arbitrary data no existence result is known.

The plan of this paper is as follows: In $\S 2$ we deduce the linear estimates to be used in the proof of our nonlinear result. Section 3 is concerned with estimates involving fractional derivatives. The proof of these estimates will be given in [26]. In $\S 4$ we shall prove Theorem 1.2. Finally, Theorem 1.3 and Corollary 1.4 are established in $\S 5$.

\section{Notations.}

$H f(x)$ denotes the Hilbert transform of $f$, i.e., $H f(x)=$ p.v. $\frac{1}{x} * f=$ $(i \operatorname{sgn}(\xi) \hat{f}(\xi))^{\vee}$. 
$D_{x}=\left(-\partial_{x}^{2}\right)^{1 / 2}$ and $\tilde{\bar{D}}_{x}^{\alpha}=H \circ D_{x}^{\alpha}$.

$L_{s}^{p}(\mathbb{R})=\left(1-\partial_{x}^{2}\right)^{-s / 2} L^{p}(\mathbb{R})$.

$H_{\text {loc }}^{s}(\mathbb{R})$ : the space of functions $h$ such that if $\phi \in C_{0}^{\infty}(\mathbb{R})$ then $\phi h \in$ $H^{s}(\mathbb{R})$.

For $g: \mathbb{R} \times[-T, T] \rightarrow \mathbb{R}$ (or $\mathbb{C})$

$$
\|g\|_{L_{x}^{p} L_{T}^{q}}=\left(\int_{-\infty}^{\infty}\left(\int_{-T}^{T}|g(x, t)|^{q} d t\right)^{p / q} d x\right)^{1 / p}
$$

in the case $T=\infty$ we shall use $\|\cdot\|_{L_{x}^{p} L_{i}^{q}}$.

$C_{\omega}(I: B):$ the space of all weakly continuous functions on an interval $I$ into a Banach space $B$.

$C_{b}(\mathbb{R}: B)=C(\mathbb{R}: B) \cap L^{\infty}(\mathbb{R}: B)$.

\section{LINEAR ESTIMATES}

In this section several estimates concerning the unitary group $\{V(t)\}_{-\infty}^{\infty}$ shall be deduced. As commented above these will be the main tools in the proof of the nonlinear results in $\S 4$. Thus we consider the linear IVP

$$
\begin{gathered}
\partial_{t} v-\partial_{x} D_{x} v=0, \quad t, x \in \mathbb{R}, \\
v(x, 0)=v_{0}(x),
\end{gathered}
$$

and

$$
\begin{gathered}
\partial_{t} w-\partial_{x} D_{x} w=f(x, t), \quad t, x \in \mathbb{R}, \\
w(x, 0)=0,
\end{gathered}
$$

whose solutions can be described by the group $\{V(t)\}_{-\infty}^{\infty}$, i.e.,

$$
v(x, t)=V(t) v_{0}(x), \quad w(x, t)=\int_{0}^{t} V(t-\tau) f(\cdot, \tau) d \tau
$$

where $V(t) v_{0}(x)=\left(S_{t} * v_{0}\right)(x)$ and

$$
S_{t}(x)=c \int_{-\infty}^{\infty} e^{i x \xi} e^{-i \xi|\xi| t} d \xi
$$

First we have sharp versions of the smoothing effect of Kato type described in the introduction.

Theorem 2.1. There exists a constant $c_{0}, c_{1}$ such that

$$
\left(\int_{-\infty}^{\infty}\left|D_{x}^{1 / 2} V(t) v_{0}(x)\right|^{2} d t\right)^{1 / 2}=c_{0}\left\|v_{0}\right\|_{2}
$$

for any $x \in \mathbb{R}$.

$$
\left\|D_{x}^{1 / 2} \int_{-\infty}^{\infty} V(t) g(\cdot, t) d t\right\|_{2} \leq c_{1} \int_{-\infty}^{\infty}\left(\int_{-\infty}^{\infty}|g(x, t)|^{2} d t\right)^{1 / 2} d x
$$

and

$$
\begin{gathered}
\sup _{x}\left(\int_{-\infty}^{\infty}\left|D_{x}\left(\int_{0}^{t} V(t-\tau) f(\cdot, \tau) d \tau\right)\right|^{2} d t\right)^{1 / 2} \\
\leq c_{1} \int_{-\infty}^{\infty}\left(\int_{-\infty}^{\infty}|f(x, t)|^{2} d t\right)^{1 / 2} d x
\end{gathered}
$$


Proof. For the proof of (2.3) we refer to [23, Theorem 4.1] (see also [24, Lemma 2.1]). For previous estimates of $L_{\text {loc }}^{2} L_{\text {loc }}^{2}$ type we refer to [13, 33, 42].

(2.4) follows from (2.3) by duality.

To obtain (2.5) we take Fourier transform in both variables in (2.2). Without loss of generality we shall assume $f \in S\left(\mathbb{R}^{2}\right)$. Thus formally we have

$$
D_{x} w(x, t)=c \int_{-\infty}^{\infty} \int_{-\infty}^{\infty} e^{i t \tau} e^{i x \xi} \frac{|\xi|}{\tau-\xi|\xi|} \hat{f}(\xi, \tau) d \xi d \tau
$$

Using Plancherel's theorem one sees that

$$
\begin{aligned}
\left(\int_{-\infty}^{\infty}\left|D_{x} w(x, t)\right|^{2} d t\right)^{1 / 2} & =c\left(\int_{-\infty}^{\infty}\left|\int_{-\infty}^{\infty} e^{i x \xi} \frac{|\xi|}{\tau-\xi|\xi|} \hat{f}(\xi, \tau) d \xi\right|^{2} d \tau\right)^{1 / 2} \\
& =c\left(\int_{-\infty}^{\infty}\left|\int_{-\infty}^{\infty} K(x-y, \tau) \hat{f}^{(t)}(y, \tau) d y\right|^{2} d \tau\right)^{1 / 2}
\end{aligned}
$$

with $\hat{f}^{(t)}$ denoting the Fourier transform of $f$ in the time variable and $K(l, \tau)$ the inverse Fourier transform (in the space variable) of the temperate distribution defined as the principal value of $|\xi| /(\tau-\xi|\xi|)$. Hence for $\tau>0$

$$
K(l, \tau)=c \int_{-\infty}^{\infty} e^{i l \sqrt{\tau} \eta} \frac{|\eta|}{1-\eta|\eta|} d \eta .
$$

In a neighborhood of the singular points $\eta=1$ and $\eta= \pm \infty$ the function $|\eta|(1-\eta|\eta|)^{-1}$ behaves like the kernel of the Hilbert transform $1 / \eta$ (or its translates) whose Fourier transform is $c \operatorname{sgn}(y)$. Thus a comparison argument shows that for $\tau>0 K(l, \tau)$ is bounded. Since $K(l, \tau)=-K(-l,-\tau)$ we conclude that $K \in L^{\infty}(\mathbb{R} \times \mathbb{R})$ with norm $M$.

Combining this bound with Minkowski's inequality, Plancherel's theorem and (2.6) it follows that

$$
\begin{gathered}
\left(\int_{-\infty}^{\infty}\left|D_{x} w(x, t)\right|^{2} d t\right)^{1 / 2}=c\left(\int_{-\infty}^{\infty}\left|\int_{-\infty}^{\infty} K(x-y, \tau) \hat{f}^{(t)}(y, \tau) d y\right|^{2} d \tau\right)^{1 / 2} \\
\leq c M \int_{-\infty}^{\infty}\left\|\hat{f}^{(t)}(y, \cdot)\right\|_{2} d y=c M \int_{-\infty}^{\infty}\left(\int_{-\infty}^{\infty}|f(y, t)|^{2} d t\right)^{1 / 2} d y
\end{gathered}
$$

which is the desired estimate. However

$$
w(x, t)=c \int_{-\infty}^{\infty} \int_{-\infty}^{\infty} e^{i t \tau} e^{i x \xi} \frac{1}{\tau-\xi|\xi|} \hat{f}(\xi, \tau) d \tau d \xi
$$

may not satisfy the initial condition in (2.2). Thus we need to consider

$$
\tilde{\bar{w}}(x, t)=w(x, t)-V(t) w(x, 0)
$$

where

$$
\begin{aligned}
w(x, 0) & =c \int_{-\infty}^{\infty} e^{i x \xi}\left(\int_{-\infty}^{\infty} \frac{1}{\tau-\xi|\xi|} \hat{f}(\xi, \tau) d \tau\right) d \xi \\
& =c \int_{-\infty}^{\infty} e^{i x \xi}\left(\int_{-\infty}^{\infty} \hat{f}^{(x)}(\xi, s) \operatorname{sgn}(s) e^{i s \xi|\xi|} d s\right) d \xi \\
& =\int_{-\infty}^{\infty} V(s) \operatorname{sgn}(s) f(x,-s) d s .
\end{aligned}
$$


From (2.4) we infer that

$$
D_{x}^{1 / 2} w(x, 0) \in L^{2}(\mathbb{R}),
$$

and by (2.3) that $\widetilde{\bar{w}}(x, t)$ the solution of (2.2) defined in (2.7) satisfies the estimate (2.5). For a more detailed proof we refer to [26].

To interpolate the estimates $(2.3)-(2.4)$ we shall need the following version of them.

\section{Corollary 2.2.}

$$
\left(\int_{-\infty}^{\infty}\left|D_{x}^{1 / 2+i \alpha} V(t) v_{0}(x)\right|^{2} d t\right)^{1 / 2}=c_{0}\left\|v_{0}\right\|_{2}
$$

for any $x \in \mathbb{R}$ with $c_{0}$ independent of $\alpha$ and

$$
\begin{gathered}
\sup _{x}\left(\int_{-\infty}^{\infty}\left|D^{1+i \alpha}\left(\int_{0}^{t} V(t-\tau) f(\cdot, \tau) d \tau\right)\right|^{2} d t\right)^{1 / 2} \\
\leq c_{1}(\alpha) \int_{-\infty}^{\infty}\left(\int_{-\infty}^{\infty}|f(x, t)|^{2} d t\right)^{1 / 2} d x
\end{gathered}
$$

where $c_{1}(\alpha)$ depends on $\alpha$ but $c_{1}(\alpha)=O\left(|\alpha|^{k}\right)$ (for some $k \in \mathbb{N}$ ) as $|\alpha|$ tends to infinity.

Proof. (2.8) follows directly from (2.3).

To prove (2.9) as in (2.6) for $\tau>0$ we consider

$$
K_{\alpha}(l, \tau)=(\sqrt{\tau})^{i \alpha} \int_{-\infty}^{\infty} e^{i l \sqrt{\tau} \eta} \frac{|\eta|^{1+i \alpha}}{1-\eta|\eta|} d \eta
$$

A combination of the result 6.11 in $[34$, p. 51] with the comparison argument used in the proof of $(2.5)$ shows that $K_{\alpha}(l, \tau) \in L^{\infty}\left(\mathbb{R}^{2}\right)$ with the norm depending on $\alpha$ in the appropriate manner. Once this bound has been established the rest of the proof follows the argument used to obtain (2.5).

Next we state the generalized one-dimensional version of a Strichartz estimate in [37] due to Ginibre and Velo [17].

Theorem 2.3. Let $p \in[2, \infty]$ and $q$ be such that $2 / q=1 / 2-1 / p$. Then

$$
\left(\int_{-\infty}^{\infty}\left\|V(t) v_{0}\right\|_{p}^{q} d t\right)^{1 / q} \leq c\left\|v_{0}\right\|_{2}
$$

and

$$
\left(\int_{-\infty}^{\infty}\left\|\int_{0}^{t} V(t-\tau) f(\cdot, \tau) d \tau\right\|_{p}^{q} d t\right)^{1 / q} \leq C\left(\int_{-\infty}^{\infty}\|f(\cdot, t)\|_{p^{\prime}}^{q^{\prime}} d t\right)^{1 / q^{\prime}}
$$

where $1 / p+1 / p^{\prime}=1 / q+1 / q^{\prime}=1$.

Proof. (See [17, p. 377].)

To complement the previous estimates we need to consider the maximal function associated with the group $\{V(t)\}_{-\infty}^{\infty}$ i.e., $\sup _{t} V(t)$. This idea was already used (in a nonoptimal manner) by Ginibre and Tsutsumi [15] in their work on uniqueness of solutions of the generalized $\mathrm{KdV}$ equation. 
Theorem 2.4.

$$
\left(\int_{-\infty}^{\infty} \sup _{-\infty<t<\infty}\left|V(t) v_{0}(x)\right|^{4} d x\right)^{1 / 4} \leq c\left\|D_{x}^{1 / 4} v_{0}\right\|_{2},
$$

and

$$
\begin{aligned}
& \left(\int_{-\infty}^{\infty} \sup _{-\infty<t<\infty}\left|\int_{0}^{t} V(t-\tau) f(\cdot, \tau) d \tau\right|^{4} d x\right)^{1 / 4} \\
& \quad \leq c\left(\int_{-\infty}^{\infty}\left(\int_{-\infty}^{\infty}\left|D_{x}^{1 / 2} f(x, t)\right| d t\right)^{4 / 3} d x\right)^{3 / 4} .
\end{aligned}
$$

Moreover for $s>\frac{1}{2}$ and $\rho>\frac{3}{4}$

$$
\left(\int_{-\infty}^{\infty} \sup _{0 \leq t \leq T}\left|V(t) v_{0}(x)\right|^{2} d x\right)^{1 / 2} \leq c(1+T)^{p}\left\|v_{0}\right\|_{s, 2} .
$$

Proof. The estimate (2.12) is due to Kenig and Ruiz [27] (for a different proof see [23, Theorem 2.5]). It is interesting to remark that (2.12) does not hold with $D_{x}^{s}, s<\frac{1}{4}$, instead of $D_{x}^{1 / 2}$ in its right-hand side (see [27, 42]).

To obtain (2.13) one needs to combine the result in [23, Theorem $2.1(2.5)$ ] with the method used in [23, Theorem 2.5] to prove (2.12). For the details of this proof we refer to [26].

The inequality (2.14) is due to Vega [42].

Corollary 2.5. For any $\alpha \in \mathbb{R}$

$$
\left(\int_{-\infty}^{\infty} \sup _{-\infty<t<\infty}\left|D^{i \alpha} V(t) v_{0}(x)\right|^{4} d x\right)^{1 / 4} \leq c\left\|D_{x}^{1 / 4} v_{0}\right\|_{2}
$$

and

$$
\begin{gathered}
\left(\int_{-\infty}^{\infty} \sup _{-\infty<t<\infty}\left|D^{i \alpha}\left(\int_{0}^{t} V(t-\tau) f(\cdot, \tau) d \tau\right)\right|^{2} d t\right)^{1 / 2} \\
\leq c(\alpha)\left(\int_{-\infty}^{\infty}\left(\int_{-\infty}^{\infty}\left|D_{x}^{1 / 2} f(x, t)\right| d t\right)^{4 / 3} d x\right)^{3 / 4} .
\end{gathered}
$$

Proof. For the proof of (2.16) we refer to [23].

Interpolating the previous estimates via Stein's theorem on analytic families of operators [35] we obtain the inequalities to be used in $\S \S 4,5$.

Theorem 2.6. For any $\theta \in[0,1]$ and $T \in(0, \infty)$,

$$
\left\|D_{x}^{(3 \theta-1) / 4} V(t) v_{0}\right\|_{L_{x}^{4 /(1-\theta)} L_{t}^{2 / \theta}} \leq c\left\|v_{0}\right\|_{2},
$$

$$
\begin{gathered}
\left\|D_{x}^{5 \theta / 4}\left(\int_{0}^{t} V(t-\tau) f(\cdot, \tau) d \tau\right)\right\|_{L_{x}^{4 /(1-\theta)} L_{t}^{2 / \theta}} \leq c T^{(1-\theta) / 2}\left\|D_{x}^{1 / 4} f\right\|_{L_{x}^{2 /(1+\theta)} L_{T}^{2}}, \\
(2.19)\left\|D_{x}^{(3 \theta-1) / 2}\left(\int_{0}^{t} V(t-\tau) f(\cdot, \tau) d \tau\right)\right\|_{L_{x}^{4 /(1-\theta)} L_{t}^{2 / \theta}} \leq c\|f\|_{L_{x}^{4 /(3+\theta)} L_{t}^{2 /(2-\theta)}} .
\end{gathered}
$$


Moreover the estimates (2.18)-(2.19) still hold with $\widetilde{\widetilde{D}}_{x}$ instead of $D_{x}$ if one replaces $f$ by $H f$ in their right-hand sides.

We recall the notation $\|\cdot\|_{L_{x}^{p} L_{T}^{q}}$ :

$$
\|f\|_{L_{x}^{p} L_{T}^{q}} \equiv\left(\int_{-\infty}^{\infty}\left(\int_{-T}^{T}|f(x, t)|^{q} d t\right)^{p / q} d x\right)^{1 / p}
$$

for $T \in(0, \infty]$.

Proof. (2.17) follows from (2.8), (2.15), and the Three Lines Theorem [4]. Similarly, (2.18) can be deduced from (2.9), (2.15), and Hölder's inequality. Finally (2.19) can be deduced from (2.9) and (2.16). Notice that for $\theta=\frac{1}{3}$, (2.19) is equal to (2.11) with $p=q=6$.

\section{LEIBNIZ'S FORMULA AND THE CHAIN RULE}

In this section we shall state the inequalities needed in $\S \S 4$ and 5 in the proof of our nonlinear results. Roughly speaking they are vector valued versions of the scalar estimates established by Kato and Ponce [22], Christ and Weinstein [10], and Taylor [39] (for previous results in this direction see Strichartz [37, Theorem 2.1, Chapter 2]). As in those works the proof is based on ideas of Coifman and Meyer [11] and Bony [8]. In this case we shall combine them with the Hardy spaces results of Fefferman and Stein [14], the tent spaces of Coifman, Meyer, and Stein [12] and vector valued inequalities due to Benedeck, Calderon, and Panzone [2] and Rubio, Ruiz, and Torrea [31].

For the details of the proof we refer to [26].

We assume $f, g: \mathbb{R} \times[-T, T] \rightarrow R$ (or $\mathbb{C}$ ) with $T \in(0, \infty]$ and $F: \mathbb{R} \rightarrow \mathbb{R}$ (or $F: \mathbb{C} \rightarrow \mathbb{C}$ ) with $F \in C^{1}(\mathbb{R})$ and $F(0)=0$.

Lemma 3.1. Let $p, q \in(1, \infty)$ and $\alpha \in(0,1)$. Then for any $T \in(0, \infty]$

$$
\begin{aligned}
& \left\|D_{x}^{\alpha}(f g)-f D^{\alpha} g-g D^{\alpha} f\right\|_{L_{x}^{p} L_{T}^{q}} \\
& \quad \leq c\left\|D_{x}^{\alpha_{1}} f\right\|_{L_{x}^{p_{1}} L_{T}^{q_{1}}}\left\|D_{x}^{\alpha_{2}} g\right\|_{L_{x}^{p_{2}} L_{T}^{q_{2}}}
\end{aligned}
$$

where $p_{i}, q_{i} \in(1, \infty), i=1,2$, with

$$
\frac{1}{p}=\frac{1}{p_{1}}+\frac{1}{p_{2}}, \quad \frac{1}{q}=\frac{1}{q_{1}}+\frac{1}{q_{2}},
$$

and $\alpha_{i} \in[0, \alpha]$ such that $\alpha_{1}+\alpha_{2}=\alpha$.

The inequality (3.1) still holds in the cases

$$
q=1 \text { with } \alpha_{i} \in(0, \alpha)
$$

and

$$
(p, q)=(1,2)
$$

Moreover in all these cases the estimate (3.1) remains valid with $\widetilde{\bar{D}}_{x}$ instead of $D_{x}$.

Lemma 3.2. Let $p, q \in(1, \infty)$ and $\alpha \in(0,1)$. Then

$$
\left\|D_{x}^{\alpha} F(f)\right\|_{L_{x}^{p} L_{T}^{q}} \leq c\left\|F^{\prime}(f)\right\|_{L_{x}^{p_{1}} L_{T}^{q_{1}}}\left\|D_{x}^{\alpha} f\right\|_{L_{x}^{p_{2}} L_{T}^{q_{2}}}
$$


where $p_{1}, p_{2}, q_{2} \in(1, \infty), q_{1} \in(1, \infty]$ with

$$
\frac{1}{p}=\frac{1}{p_{1}}+\frac{1}{p_{2}} \text { and } \frac{1}{q}=\frac{1}{q_{1}}+\frac{1}{q_{2}} .
$$

Moreover the estimate (3.3) still holds with $\widetilde{\bar{D}}_{x}$ instead of $D_{x}$.

\section{Proof of Theorem 1.2}

For simplicity in the exposition we shall only consider the case $k \geq 4$. The proof when $k=2$ ( $k=3$ respectively) follows the same argument, but with the estimate (2.14) instead of $(2.12)$ (the $L_{x}^{3} L_{T}^{\infty}$ estimate obtained by interpolating (2.12)-(2.14) instead of (2.12), respectively).

Without loss of generality we restrict ourselves to the most interesting case $s=\frac{3}{4}$.

Thus for $u_{0} \in H^{3 / 4}(\mathbb{R})$ with $\left\|u_{0}\right\|_{3 / 4,2} \leq \delta$ (to be determined below) we define $\Phi_{u_{0}}(v)=\Phi(v)=u$ as the solutions of the linear IVP

$$
\partial_{t} u-\partial_{x} D_{x} u=-v^{k} \partial_{x} v, \quad u(x, 0)=u_{0}(x),
$$

with $v \in X_{T}^{a}$ where

$X_{T}^{a}=\left\{v: \mathbb{R} \times[-T, T] \rightarrow \mathbb{R} \mid \eta_{T}(v) \leq a, \lambda_{T, \theta}(v)+\lambda_{T, \theta}(H v) \leq a, \quad \theta \in[0,1]\right\}$ where the following notation has been introduced:

$$
\eta_{T}(v) \equiv \sup _{[-T, T]}\|v(t)\|_{3 / 4,2}
$$

and for $\theta \in[0,1]$,

$$
\lambda_{T, \theta}(v) \equiv\left\|D_{x}^{5 \theta / 4} v\right\|_{L_{x}^{4 /(1-\theta)} L_{T}^{2 / \theta}} .
$$

As mentioned above, for $T \in(0, \infty)$ and $\alpha \in[0,1]$

$$
\|f\|_{L_{x}^{p} L_{T}^{q}} \equiv\left(\int_{-\infty}^{\infty}\left(\int_{0}^{T}|f(x, t)|^{q} d t\right)^{p / q} d x\right)^{1 / p}, \quad D_{x}^{\alpha} h(x)=c\left(|\xi|^{\alpha} \hat{h}(\xi)\right)^{\vee}
$$

and

$$
\widetilde{\bar{D}}_{x}^{\alpha} h(x)=c^{\prime}\left(\operatorname{sgn}(\xi)|\xi|^{\alpha} \hat{h}(\xi)\right)^{\vee}=c^{\prime \prime} H D_{x}^{\alpha} h(x)
$$

with $H$ denoting the Hilbert transform.

Our aim is to show that there exist $a$ and $T$ (depending only on $k$ and $\left\|u_{0}\right\|_{3 / 4,2}$ in the appropriate manner) such that if $v \in X_{T}^{a}$ then $\Phi_{u_{0}}(v)=$ $\Phi(v) \in X_{T}^{a}$ and the map $\Phi_{u_{0}}: X_{T}^{a} \rightarrow X_{T}^{a}$ is a contraction.

By definition

$$
\Phi(v)(t)=\Phi_{u_{0}}(v)(t)=V(t) u_{0}-\int_{0}^{t} V(t-\tau)\left(v^{k} \partial_{x} v\right)(\tau) d \tau
$$

A combination of (2.17)-(2.18) with the notation in (4.4) yields the estimates

$$
\lambda_{T, \theta}(\Phi(v)) \leq c\left\|u_{0}\right\|_{3 / 4,2}+c T^{(1-\theta) / 2}\left\|D_{x}^{1 / 4}\left(v^{k} \partial_{x} v\right)\right\|_{L_{x}^{2 /(1+\theta)} L_{T}^{2}}
$$

and 


$$
\lambda_{T, \theta}(H \Phi(v)) \leq c\left\|u_{0}\right\|_{3 / 4,2}+c T^{(1-\theta) / 2}\left\|\tilde{\bar{D}}_{x}^{1 / 4}\left(v^{k} \partial_{x} v\right)\right\|_{L_{x}^{2 /(1+\theta)} L_{T}^{2}}
$$

for any $\theta \in[0,1]$.

Now using (3.1) with $p \in[1,2]$, Hölder's and Sobolev's inequalities we obtain the following string of inequalities:

$$
\begin{aligned}
& \left\|D_{x}^{1 / 4}\left(v^{k} \partial_{x} v\right)\right\|_{L_{x}^{p} L_{T}^{2}} \\
& \leq c\left\|D_{x}^{1 / 4}\left(v^{k} \partial_{x} v\right)-v^{k} D_{x}^{1 / 4} \partial_{x} v-D_{x}^{1 / 4}\left(v^{k}\right) \partial_{x} v\right\|_{L_{x}^{p} L_{T}^{2}}
\end{aligned}
$$

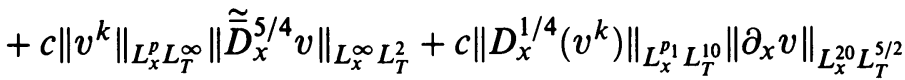

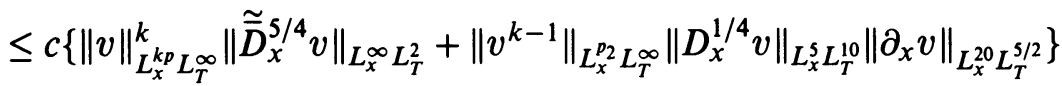

$$
\begin{aligned}
& \leq c\left\{\left(\sup _{x} \sup _{[-T, T]}|v|^{k p-4}\right)^{1 / p}\|v\|_{L_{x}^{4} L_{T}^{\infty}}^{4 / p}\left\|\tilde{\bar{D}}_{x}^{5 / 4} v\right\|_{L_{x}^{\infty} L_{T}^{5 / 2}}\right. \\
& \left.+\left(\sup _{x} \sup _{[-T, T]}|v|^{(k-1) p_{2}-4}\right)^{1 / p_{2}}\|v\|^{4 / p_{2}}\left\|_{L_{x}^{4} L_{T}^{\infty}}\right\| D_{x}^{1 / 4} v\left\|_{L_{x}^{5} L_{T}^{10}}\right\| \partial_{x} v \|_{L_{x}^{20} L_{T}^{5 / 2}}\right\} \\
& \leq c\left\{\left(\sup _{[-T, T]}\|v(t)\|_{3 / 4,2}\right)^{k-4 / p}\left(\lambda_{T, 0}(v)\right)^{4 / p} \lambda_{T, 1}\left(H_{v}\right)\right. \\
& \left.+\left(\sup _{[-T, T]}\|v(t)\|_{3 / 4,2}\right)^{k-1-4 / p_{2}}\left(\lambda_{T, 0}(v)\right)^{4 / p_{2}}\left(\lambda_{T, 1 / 5}(v)\right)\left(\lambda_{T, 4 / 5}(v)\right)\right\} \\
& \leq c\left(\Lambda_{T}(v)\right)^{k+1} \equiv D
\end{aligned}
$$

where

$$
\Lambda_{T}(v)=\max \left\{\sup _{[-T, T]}\|v(t)\|_{3 / 4,2} ; \sup _{0 \leq \theta \leq 1} \lambda_{T, \theta}(v) ; \sup _{0 \leq \theta \leq 1} \lambda_{T, \theta}(H v)\right\}
$$

and

$$
\frac{1}{p}=\frac{1}{p_{1}}+\frac{1}{20}=\frac{1}{p_{2}}+\frac{1}{5}+\frac{1}{20}
$$

A similar argument shows that for any $p \in[1,2]$

$$
\left\|\tilde{\bar{D}}_{x}^{1 / 4}\left(v^{k} \partial_{x} v\right)\right\|_{L_{x}^{p} L_{T}^{2}} \leq D
$$

From (4.6)-(4.10) one sees that

$$
C \sup _{0 \leq \theta \leq 1} \lambda_{T, \theta}(\Phi(v))+\sup _{0 \leq \theta \leq 1} \lambda_{T, \theta}(H \Phi(v)) \leq c\left\|u_{0}\right\|_{3 / 4,2}+c(1+T) D .
$$


On the other hand, inserting (2.4) in (4.5) it follows that

$$
\begin{aligned}
& \sup _{[-T, T]}\|\Phi(v)(t)\|_{3 / 4,2} \\
& \leq c \sup _{[-T, T]}\|\Phi(v)(t)\|_{2}+c \sup _{[-T, T]}\left\|D_{x}^{3 / 4} \Phi(v)(t)\right\|_{2} \\
& \leq c\left\|u_{0}\right\|_{2}+c \int_{0}^{T}\left(\int_{-\infty}^{\infty}\left|v^{k} \partial_{x} v\right|^{2} d x\right)^{1 / 2} d t+c D \\
& \leq c^{\prime}\left\|u_{0}\right\|_{2}+c T^{1 / 2}\left(\int_{-\infty}^{\infty} \int_{0}^{T}\left|v^{k} \partial_{x} v\right|^{2} d t d x\right)^{1 / 2}+c D \\
& \leq c^{\prime}\left\|u_{0}\right\|_{2}+c T^{1 / 2}\left\|v^{k}\right\|_{L_{x}^{20} L_{T}^{10}}\left\|\partial_{x} v\right\|_{L_{x}^{20 / 9} L_{T}^{5 / 2}}+c D \\
& \leq c\left\|u_{0}\right\|_{2}+c T^{3 / 5}\left(\sup _{x} \sup _{[-T, T]}|v|\right)^{k-9 / 5}\left(\lambda_{T, 0}(v)\right)^{9 / 5}\left(\lambda_{T, 4 / 5}(v)\right)+c D \\
& \leq c\left\|u_{0}\right\|_{3 / 4,2}+c^{\prime}(1+T) D .
\end{aligned}
$$

Estimates (4.6)-(4.12) lead to

$$
\Lambda_{T}(\Phi(v)) \leq c\left\|u_{0}\right\|_{3 / 4,2}+c(1+T)\left(\Lambda_{T}(v)\right)^{k+1} .
$$

Fixing

$$
a=2 c\left\|u_{0}\right\|_{3 / 4,2}=2 c \delta_{0} \leq 2 c \delta,
$$

it follows that $\Phi\left(X_{T}^{a}\right) \subseteq X_{T}^{a}$ whenever

$$
2(1+T) c^{k} \delta_{0}^{k} \leq \frac{1}{2} .
$$

The same argument used above proves that for every $v, w \in X_{T}^{a}$ with $a$ and $T$ as in (4.14)-(4.15) respectively one has

$$
\Lambda(\Phi(v)-\Phi(w)) \leq 2(1+T) c^{k} \delta_{0}^{k} \Lambda(v-2) \leq \frac{1}{2} \Lambda(v-w) .
$$

Therefore $\Phi: X_{T}^{a} \rightarrow X_{T}^{a}$ is a contraction.

Similarly we have that for $T^{\prime} \in(0, T)$ there exists $\delta_{0}^{\prime}>0$ such that if $w_{0} \in H^{3 / 4}(\mathbb{R})$ with $\left\|w_{0}-u_{0}\right\|_{3 / 4,2}<\delta_{0}^{\prime}$ there the solution $\Phi_{w_{0}}(w)=w$ of the integral equation form of the IVP (1.10) satisfies

$$
w \in X_{T^{\prime}}^{a}
$$

and

$$
\Lambda(u-w) \leq c\left\|u_{0}-w_{0}\right\|_{3 / 4,2} .
$$

Thus we have proven that there exists $\delta>0$ such that for $u_{0} \in H^{3 / 4}(\mathbb{R})$ with $\left\|u_{0}\right\|_{3 / 4,2}<\delta$ the integral equation

$$
u(t)=V(t) u_{0}-\int_{0}^{t} V(t-\tau)\left(u^{k} \partial_{x} u\right)(\tau) d \tau
$$

has a unique solution in the class $X_{T}^{a}$ with $T=T\left(\left\|u_{0}\right\|_{3 / 4,2} ; k\right)>0$. Furthermore the map $\tilde{\bar{u}}_{0} \rightarrow \tilde{\bar{u}}(t)$ is locally Lipschitz. 
To complete the proof of Theorem 1.2 we shall establish the persistence property (i.e., $\left.u \in C\left([-T, T]: H^{3 / 4}\right)\right)$ and the uniqueness result in a class larger than $X_{T}^{a}$, i.e., $X_{T}^{\infty}$.

It suffices to show that the map $t \rightarrow u(t)$ from $[-T, T]$ into $H^{3 / 4}(\mathbb{R})$ is continuous at zero.

Using the smallness assumption on the data $u_{0}$ and the estimates (4.6)-(4.7) (with $\theta=1$ ) and (4.8) it follows that

$$
\lambda_{T^{\prime}, 1}(u)+\lambda_{T^{\prime}, 1}(H u) \leq 2 c \lambda_{T^{\prime}, 1}\left(V(t) u_{0}\right)+o(1)
$$

as $T^{\prime}$ tends to zero.

A simple argument shows that $\lambda_{T^{\prime}, 1}\left(V(t) u_{0}\right)=o(1)$ as $T^{\prime}$ tends to zero, therefore

$$
\lambda_{T^{\prime}, 1}(u)+\lambda_{T^{\prime}, 1}(H u)=o(1)
$$

as $T^{\prime}$ tends to zero. Finally using (4.20) in the integral equation

$$
u(t)-u_{0}=V(t) u_{0}-u_{0}-\int_{0}^{t} V(t-\tau)\left(u^{k} \partial_{x} u\right)(\tau) d \tau
$$

together with the group properties and the estimates (4.12)-(4.8) we obtain the desired result.

Now let $\widetilde{\widetilde{u}} \in X_{T^{\prime}}^{a^{\prime}} \cap C\left([-T, T]: H^{3 / 4}\right)$ with $a^{\prime}>a$ be a solution of the integral equation (4.19) in the time interval $\left[-T_{0}, T_{0}\right]$ where $T_{0}=\min _{\left\{T ; T^{\prime}\right\}}$. By continuity and (4.14) there exists $T_{1} \in\left(0, T_{0}\right)$ such that $\sup _{\left[-T_{1}, T_{1}\right]}\|\tilde{\bar{u}}(t)\|_{3 / 4,2} \leq a$.

A similar argument to that used in (4.20) shows that

$$
\lambda_{T^{\prime}, \theta}(\tilde{\bar{u}})+\lambda_{T^{\prime}, \theta}(H \tilde{\bar{u}})=o(1)
$$

as $T^{\prime}$ tends to zero (uniformly in $\theta \in[0,1]$ ). Therefore for sufficiently small $T_{2}$ it follows that $\tilde{\bar{u}} \in X_{T_{2}}^{a}$ which implies that $u \equiv \tilde{\bar{u}}$ in $\mathbb{R} \times\left[-T_{2}, T_{2}\right]$. Reapplying this argument we extend this uniqueness result to the time interval $[-T, T]$.

\section{Proofs of Theorem 1.3 and Corollary 1.4}

The proof of Theorem 1.3 is similar to that explained in the previous section for Corollary 1.4. In fact, since local existence is known (Theorem 1.2) one just needs to sketch the argument used to obtain an a priori estimate which allows us to extend the local solution to a global one.

We shall restrict ourselves to the most interesting case $s=1$. Thus we introduce the space

$$
Z_{a}=\left\{v: \mathbb{R} \times \mathbb{R} \rightarrow \mathbb{R} \mid \eta(v) \leq a, \sup _{0 \leq \theta \leq 1}\left(\nu_{\theta}(v)+\nu_{\theta}(H v)\right) \leq a \text { and } \beta(v) \leq a\right\}
$$

where

$$
\eta(v)=\sup _{t}\|v(t)\|_{1,2}, \quad \nu_{\theta}(v)=\left\|D_{x}^{3 \theta / 2} v\right\|_{L_{x}^{4 /(1-\theta)} L_{t}^{2 / \theta}}
$$

and

$$
\beta(v)=\left(\int_{-\infty}^{\infty}\|v(\cdot, t)\|_{\infty}^{4} d t\right)^{1 / 4} .
$$


By Theorem 1.2 there exists $u(\cdot)$ defining in the time interval $[-T, T]$ such that

$$
u(t)=V(t) u_{0}-\int_{0}^{t} V(t-\tau)\left(u^{k} \partial_{x} u\right)(\tau) d \tau
$$

From (2.10) with $p=\infty$ we find the a priori estimate

$$
\begin{aligned}
\beta(u) & \leq c\left\|u_{0}\right\|_{2}+c \int_{-\infty}^{\infty}\left\|\left(u^{k} \partial_{x} u\right)(\tau)\right\|_{2} d \tau \\
& \leq c\left\|u_{0}\right\|_{2}+c \sup _{t}\|u(t)\|_{1,2}^{k-3} \int_{-\infty}^{\infty}\|u(\cdot, t)\|_{\infty}^{4} d t \\
& \leq c\left\|u_{0}\right\|_{2}+c(\eta(u))^{k-3}(\beta(u))^{4} .
\end{aligned}
$$

More generally, the inequalities (2.17)-(2.19) together with the notation

$$
\Lambda(v)=\sup \left\{\eta(v) ; \sup _{0 \leq \theta \leq 1}\left(\nu_{\theta}(v)+\nu_{\theta}(H(v)) ; \beta(v)\right\}\right.
$$

and the argument used in the previous section yield the a priori estimate

$$
\Lambda(u) \leq c\left\|u_{0}\right\|_{1,2}+c(\Lambda(u))^{k+1} .
$$

Hence, there exists $\delta>0$ and $M>0$ such that for $u_{0} \in H^{1}(\mathbb{R})$ with $\left\|u_{0}\right\|_{1,2}<\delta$ one has that

$$
\Lambda(u) \leq M
$$

Once that the formal estimate (5.3) has been established the rest of the proof of Theorem 1.3 uses a combination of standard arguments and those in the previous section. Therefore it will be omitted.

Finally we consider Corollary 1.4. First we shall prove that

$$
\lim _{t \rightarrow \pm \infty} \int_{0}^{t} V(-\tau)\left(u^{k} \partial_{x} u\right)(\tau) d \tau
$$

exists in the $H^{1}$-norm. Thus

$$
\begin{aligned}
& \left\|\int_{t^{\prime}}^{t} V(-\tau)\left(u^{k} \partial_{x} u\right)(\tau) d \tau\right\|_{2} \leq \int_{t^{\prime}}^{t}\left\|u^{k} \partial_{x} u(\tau)\right\|_{2} d \tau \\
& \quad \leq c \sup _{\left(t, t^{\prime}\right)}\|u(t)\|_{1,2}^{k-3} \int_{t^{\prime}}^{t}\|u(\tau)\|_{\infty}^{4} d \tau \equiv E\left(t, t^{\prime}\right) .
\end{aligned}
$$

From $(1.7 \mathrm{a})-(1.7 \mathrm{~b})$ it follows that $E\left(t, t^{\prime}\right)$ tends to zero as $t, t^{\prime}$ tend to $\pm \infty$. Now combining (2.4) with (3.1), (3.2) we obtain the following string of inequalities: 


$$
\begin{aligned}
& \left\|\partial_{x} \int_{t^{\prime}}^{t} V(-\tau)\left(u^{k} \partial_{x} u\right)(\tau) d \tau\right\|_{2} \\
& =\left\|D_{x}^{1 / 2} \int_{t^{\prime}}^{t} V(-\tau) \widetilde{\bar{D}}_{x}^{1 / 2}\left(u^{k} \partial_{x} u\right)(\tau)\right\|_{2} \leq c \int_{-\infty}^{\infty}\left(\int_{t^{\prime}}^{t}\left|\tilde{\bar{D}}_{x}^{1 / 2}\left(u^{k} \partial_{x} u\right)\right|^{2} d \tau\right)^{1 / 2} d x \\
& \leq c \int_{-\infty}^{\infty}\left(\int_{t^{\prime}}^{t}\left|u^{k} D_{x}^{3 / 2} u\right|^{2} d \tau\right)^{1 / 2} d x+c \int_{-\infty}^{\infty}\left(\int_{t^{\prime}}^{t}\left|\widetilde{D}_{x}^{1 / 2}\left(u^{k}\right) \partial_{x} u\right|^{2} d \tau\right)^{1 / 2} d x \\
& +c\left(\int_{-\infty}^{\infty}\left(\int_{t^{\prime}}^{t}\left|\tilde{\bar{D}}_{x}^{1 / 4}\left(u^{k}\right)\right|^{12} d \tau\right)^{2 / 23} d x\right)^{23 / 24} \\
& \cdot\left(\int_{-\infty}^{\infty}\left(\int_{t^{\prime}}^{t}\left|\tilde{\bar{D}}_{x}^{5 / 4} u\right|^{12 / 5} d \tau\right)^{10} d x\right)^{1 / 24} \\
& \leq c\left(\int_{-\infty}^{\infty} \sup _{\left(t, t^{\prime}\right)}|u(x, \tau)|^{4} d x\right) \sup _{\left(t, t^{\prime}\right)}\|u(t)\|_{1,2}^{k-4} \sup _{x}\left(\int_{t^{\prime}}^{t}\left|\tilde{\bar{D}}_{x}^{3 / 2} u\right|^{2} d \tau\right)^{1 / 2} \\
& +c\left(\int_{-\infty}^{\infty} \sup _{\left(t, t^{\prime}\right)}|u(x, \tau)|^{4} d x\right)^{3 / 4} \sup _{\left(t, t^{\prime}\right)}\|u(t)\|_{1,2}^{k-4} \\
& \left\{\left(\int_{-\infty}^{\infty} \int_{t^{\prime}}^{t}\left|\tilde{\bar{D}}_{x}^{1 / 2} u\right|^{6} d \tau d x\right)^{1 / 6}\left(\int_{-\infty}^{\infty}\left(\int_{t^{\prime}}^{t}\left|\partial_{x} u\right|^{3} d \tau\right)^{4} d x\right)^{1 / 12}\right. \\
& +\left(\int_{-\infty}^{\infty}\left(\int_{t^{\prime}}^{t}\left|\tilde{\bar{D}}_{x}^{1 / 4} u\right|^{12} d \tau\right)^{2 / 5} d x\right)^{5 / 24} \\
& \left.-\left(\int_{-\infty}^{\infty}\left(\int_{t^{\prime}}^{t}\left|\tilde{\bar{D}}_{x}^{5 / 4} u\right|^{12 / 5} d \tau\right)^{10} d x\right)^{1 / 24}\right\}
\end{aligned}
$$

Thus from (5.3) and an argument similar to that used in (4.20) we can conclude the

$$
\left\|\partial_{x} \int_{t^{\prime}}^{t} V(-\tau)\left(u^{k} \partial_{x} u\right)(\tau) d \tau\right\|_{2}
$$

tends to zero as $t, t^{\prime}$ tend to $\pm \infty$. Hence (5.4) has been established.

Defining

$$
\omega_{0+}=u_{0}+\int_{0}^{\infty} V(-\tau)\left(u^{k} \partial_{x} u\right)(\tau) d \tau
$$

and

$$
\omega_{0-}=u_{0}+\int_{-\infty}^{0} V(-\tau)\left(u^{k} \partial_{x} u\right)(\tau) d \tau
$$

the same argument given for (5.4) yields (1.8) (for details see [36]).

\section{ACKNOWLEDGMENT}

C. E. Kenig and G. Ponce were supported by NSF grants, while L. Vega was supported by a DGICYT grant. 


\section{REFERENCES}

1. L. Abdelouhab, J. L. Bona, M. Felland, and J.-C. Saut, Nonlocal models for nonlinear dispersive waves, Phys. D 40 (1989), 360-392.

2. A. Benedek, A. P. Calderón, and R. Panzone, Convolution operators on Banach space valued functions, Proc. Nat. Acad. Sci. U.S.A. 48 (1962), 356-365.

3. T. B. Benjamin, Internal waves of permanent form in fluids of great depth, J. Fluid Mech. 29 (1967), 559-592.

4. J. Bergh and J. Löfsthöm, Interpolation spaces, Springer-Verlag, New York and Berlin, 1970.

5. T. L. Bock and M. D. Khuskal, A two parameter Miura transformation of the Benjamin-Ono equation, Phys. Lett. A 74 (1976), 173-176.

6. J. L. Bona, Private communication.

7. J. L. Bona, P. E. Souganidis and W. A. Strauss, Stability and instability of solitary waves of Korteweg-de Vries Type, Proc. Roy. Soc. London Ser. A 411 (1987), 395-412.

8. J. M. Bony, Calcul symbolique et propagation des singularités pour les équations aux dérivées partielles non linéaires, Ann. Sci. École Norm. Sup. (4) 14 (1981), 209-246.

9. K. M. Case, Benjamin-Ono related equations and their solutions, Proc. Nat. Acad. Sci. U.S.A. 76 (1976), 1-3.

10. F. M. Christ and M. I. Weinstein, Dispersion of small amplitude solutions of the generalized Korteweg-de Vries equation, J. Funct. Anal. 100 (1991), 87-109.

11. R. R. Coifman and Y. Meyer, Au delá des opérators pseudodifférentieles, Asterisque no. 57, Soc. Math. France 1973.

12. R. R. Coifman, Y. Meyer, and E. M. Stein, Some new function spaces and their applications to harmonic analysis, J. Funct. Anal. 62 (1985), 304-335.

13. P. Constantin and J. C. Saut, Local smoothing properties of dispersive equations, J. Amer. Math. Soc. 1 (1989), 413-446.

14. C. Fefferman and E. M. Stein, $H^{p}$ spaces of several variables, Acta Math. 129 (1972), 137-193.

15. J. Ginibre and Y. Tsutsumi, Uniqueness for the generalized Korteweg-de Vries equations, SIAM J. Math. Anal. 20 (1989), 1388-1425.

16. J. Ginibre and G. Velo, Smoothing properties and existence of solutions for the generalized Benjamin-Ono equation, J. Differential Equations 93 (1991), 150-212.

17. _ Scattering theory in the energy space for a class of nonlinear Schrödinger equations, J. Math. Pures Appl. 64 (1985), 363-401.

18. R. J. Iorio, On the Cauchy problem for the Benjamin-Ono equation, Comm. Partial Differential Equations 11 (1986), 1031-1081.

19. T. Kato, Quasilinear equations of evolutions, with applications to partial differential equation, Lecture Notes in Math., vol. 448, Springer-Verlag, Berlin and New York, 1975, pp. 27-50.

20. _, On the Cauchy problem for the (generalized) Korteweg-de Vries equation, Adv. Math. Suppl. Stud., Stud. Appl. Math. 8 (1983), 93-128.

21. _ Weak solutions of infinite-dimensional Hamiltonian system, preprint.

22. T. Kato and G. Ponce, Commutator estimates and the Euler and Navier-Stokes equations, Comm. Pure Appl. Math. 41 (1988), 891-907.

23. C. E. Kenig, G. Ponce, and L. Vega, Oscillatory integrals and regularity of dispersive equations, Indiana Univ. Math. J. 40 (1991), 33-69.

24. __ Well-posedness of the initial value problem for the Korteweg-de Vries equation, J. Amer. Math. Soc. 4 (1991), 323-347.

25. __ Small solutions to nonlinear Schrödinger equations, Ann. Inst. Henri Poincaré 110 (1993), 255-288.

26. __ Well-posedness and scattering results for the generalized Korteweg-de Vries equation via contraction principle, Comm. Pure Appl. Math. 46 (1993), 527-620. 
27. C. E. Kenig and A. Ruiz, A strong type $(2,2)$ estimate for the maximal function associated to the Schrödinger equation, Trans. Amer. Math. Soc. 230 (1983), 239-246.

28. H. Ono, Algebraic solitary waves in stratified fluids, J. Phys. Soc. Japan 39 (1975), 10821091.

29. G. Ponce, Smoothing properties of solutions to the Benjamin-Ono equation, Lecture Notes in Pure and Appl. Math., vol. 122 (C. Sadosky, ed.), Dekker, New York, 1990, pp. 667-679.

30. __ On the global well-posedness of the Benjamin-Ono equation, Differential Integral Equations 4 (1991), 527-542.

31. J. L. Rubio De Francia, F. J. Ruiz, and J. L. Torrea, Calderón-Zygmund theory for operatorvalued kernels, Adv. Math. 62 (1988), 7-48.

32. J.-C. Saut, Sur quelques généralisations de $I^{2}$ équations de Korteweg-de Vries, J. Math. Pures Appl. 58 (1979), 21-61.

33. P. Sjölin, Regularity of solutions to the Schrödinger equations, Duke Math. J. 55 (1987), 699-715.

34. E. M. Stein, Singular integrals and differentiability properties of functions, Princeton Univ. Press Princeton, NJ, 1970.

35. E. M. Stein and G. Weiss, Introduction to Fourier analysis in Euclidean spaces, Princeton Univ. Press, Princeton, NJ, 1971.

36. W. A. Strauss, Nonlinear scattering at low energy, J. Funct. Anal. 41 (1981), 110-133.

37. R. S. Strichartz, Multipliers in fractional Sobolev spaces, J. Math. Mech. 16 (1967), 10311060.

38. _ Restriction of Fourier transforms to quadratic surface and decoy of solutions of wave equations, Duke Math. J. 44 (1977), 705-714.

39. M. E. Taylor, Pseudo-differential operators and nonlinear P.D.E., preprint.

40. M. M. Tom, Smoothing properties of some weak solutions of the Benjamin-Ono equation, Differential Integral Equations 3 (1990), 683-694.

41. M. Tsutsumi and I. Fukuda, On solutions of the derivative nonlinear Schrödinger equation. II, Funkcial. Ekvac. 24 (1981), 85-94.

42. L. Vega, Doctoral thesis, Univ. Autonoma de Madrid, Spain, 1987.

43. __ The Schrödinger equation: pointwise convergence to the initial data, Proc. Amer. Math. Soc. 102 (1988), 874-878.

44. M. I. Weinstein, On the solitary traveling wave of generalized Korteweg-de Vries equation, Lectures in Appl. Math., vol. 23, Amer. Math. Soc., Providence, RI, 1986, pp. 23-29.

Department of Mathematics, University of Chicago, Chicago, Illinois 60637 93106

Department of Mathematics, University of California, Santa Barbara, California

Facultad de Ciencias, Universidad Autonoma de Madrid, Cantoblanco, Madrid 28049, SPAIN 